\title{
High-Q $(>750,000)$ photonic crystal nanocavities fabricated from chalcogenide glass fully embedded in an index-matched cladding
}

\author{
Xin Gai, Barry Luther-Davies*, Thomas P. White
}

Centre for Ultrahigh bandwidth Devices for Optical Systems, Laser Physics Centre

Research School of Physics and Engineering, The Australian National University, Canberra

ACT2600, Australia

\begin{abstract}
We have fabricated a $\mathrm{Ge}_{11.5} \mathrm{As}_{24} \mathrm{Se}_{64.5}$ 2-D photonic crystal containing a hetero-structure cavity fully embedded in a cladding with index of 1.44 . Because of the low index contrast of this structure $(\approx 1.2)$ we had to use a W0.54 defect waveguide to inhibit losses to continuum modes above the light line. By has allowed optical cavities with very high Q $(>750,000)$ to be obtained.
\end{abstract}

Keywords: Photonic crystals, heterostructure cavities, resonators

*barry.luther-davies@anu.edu.au; Phone +61-2-6125-4255; Fax +61-2-6125-0029; physics.anu.edu.au

\section{INTRODUCTION}

Slab photonic crystal $(\mathrm{PhC})$ waveguides, which utilize the band gap of a periodic structure to confine light in the horizontal direction whilst guiding light vertically by total internal reflection, have great potential for all-optical processing. In particular their small footprint makes them easy to integrate to create compact devices whilst their high efficiency of light confinement leads to high light intensities that enhance the nonlinear response [1,2].

Slow light propagation is one of two approaches for further enhancing the light intensity in a 2-D photonic crystal waveguide. By carefully adjusting the physical structure of the $\mathrm{PhC}$, a large group index, $n_{g}$, of several tens to about one hundred can be engineered thereby achieving slow propagation of the pulse envelope. As a consequence, the propagating pulses are compressed in space leading to an enhancement of the light intensity [3-5]. Slow light has been applied to optical processing as an optical buffer utilizing the large group index [3, 6-9]; and nonlinear processing has been observed in slow light waveguides made from highly nonlinear materials utilizing third harmonic generation for alloptical pulse monitoring [10-12]; self-phase modulation [13]; and FWM [14-17].

The second way of enhancing the nonlinear response is to incorporate a photonic crystal micro-cavity and this is particularly useful for compact switching devices operating with exceptionally low power. It is this second opportunity that we explore in this work. Of course, high quality photonic crystal resonant cavities have already been reported in silicon membranes achieving Q factors ranging from $10^{5}$ to $10^{6}$. Examples are the "L3" micro-cavities created by removing 3 holes from a periodic structure [18] and hetero-structures that create mirrors by shifting the dispersion curve for the guided mode by varying the lattice constant along the waveguide [19]. One of the most interesting phenomena achievable in a photonic crystal resonant cavity is bistable switching which would be a very useful for all-optical signal processing at low powers.

Much effort have been expended to generate high Q resonant cavities with the minimum effective volume which theoretically can reduce thermal effects in the cavities leading to ultra-fast bistable switching by the Kerr effect alone $[18,20]$. However, so far most experiments on switching have been dominated by thermal nonlinearities [21,22], or those induced by two-photon absorption and free carrier generation [23,24] and thus have slower recovery times. The fastest bistable switching that has been achieved in a silicon photonic crystal, in fact, used free carrier nonlinearities and had a relaxation time of over 100ps which is far below the requirement for ultrafast all-optical processing [23,24]. It must be noted that resonant cavities inevitably reduce the response time even for ultrafast Kerr switching, however by choosing a moderate $\mathrm{Q}(<10,000)$ switching on the $<20$ ps timescale should still be achievable.

Thus, we have been seeking solutions for photonic crystals that can decrease the role of thermal, TPA and FCA nonlinear effects with the aim of achieving switching from the Kerr nonlinearity alone. As a semiconductor, silicon shows significant TPA and FCA effects and hence our approach has been to replace the silicon with a chalcogenide glass in which TPA and FCA can be negligible. The magnitude of the thermal nonlinear effect, however, is determined by the

Photonic Crystal Materials and Devices X, edited by Hernán Ruy Míguez, Sergei G. Romanov,

Lucio Claudio Andreani, Christian Seassal, Proc. of SPIE Vol. 8425, 842516 · C 2012 SPIE

CCC code: $0277-786 X / 12 / \$ 18 \cdot$ doi: $10.1117 / 12.921526$

Proc. of SPIE Vol. $8425842516-1$ 
linear absorption in the photonic crystal waveguide and the thermo-optic coefficient, $d n / d t$, of the material. Because the thermo-optic coefficient of the chalcogenides is similar to silicon, it is difficult to reduce the thermal nonlinearity by simply changing the slab material. However, in most previous work, the photonic crystal structures have been fabricated as air-suspended membranes to reduce coupling between the guided modes and the light line by maximizing the index contrast to increase the bandwidth of the guided modes [1,2]. However, air has very poor thermal conduction leading a low threshold for the thermal nonlinearity and a very slow recovery time for thermal switching. As a result, we need to design a structure that has much better cooling since this will increase the threshold for the thermal nonlinearity.

Thus, although we cannot reduce $d n / d t$ by changing the slab material, we can reduce the temperature rise, $\Delta T$, by improving heat conduction from the slab, for example, by applying a solid cladding. Using this approach we also have the opportunity to make an a-thermal structure if we can find a cladding whose thermo-optic coefficient has the opposite sign and an appropriate magnitude compared with the slab. For example, most polymers or liquids have a negative thermo-optic coefficient compared with chalcogenide glasses for which it is positive.

Unfortunately because the chalcogenide glass has a smaller refractive index than silicon, the addition of a cladding reduces the index contrast drastically and this severely restricts the bandwidth of the guided modes under the light line. This makes it difficult to find a structure that can support a high Q resonance. Another challenge is that the fabrication process for chalcogenide glass photonic crystals is not as mature as for silicon and is difficult because the glass is relatively soft and fragile.

In this paper, we summarize work on the fabrication of high quality photonic crystals from chalcogenide glasses using electron-beam lithography and dry etching. To overcome the restriction of the limited index contrast in a fully clad chalcogenide membrane, we have designed a special structure that achieves resonances with very high Q-factors in a $\mathrm{PhC}$ slab made from a photo-stable chalcogenide glass, $\mathrm{Ge}_{11.5} \mathrm{As}_{24} \mathrm{Se}_{64.5}$, completely embedded in a cladding with the index of silica. According to our simulations a Q-factor of over 20 million could be achieved using this design. We successfully fabricated $\mathrm{PhC}$ heterostructure resonators achieving an intrinsic $\mathrm{Q}$ factor of $>750,000$ which compares favourably with a value of 2,000,000 predicted from modeling of a completely ideal structure. Thus, we have demonstrated that it is possible to obtain high-Q with a fully clad chalcogenide photonic crystal leading to a reduced thermal nonlinearity that should make observation of ultra-fast Kerr nonlinear bistable switching possible.

\section{PHC DESIGN}

In order to find a proper structure, we used the plane wave expansion method to calculate the band gap and guided modes of a W1 waveguide. As shown in Fig. 1a, the even mode exhibits a band gap between $0.269 \omega \mathrm{\omega} / \pi \mathrm{c}$ and $0.314 \omega a / \pi c$, where $a$ is the lattice constant of photonic crystal. We then calculated the guided modes for a W1 waveguide in this structure and these are shown in Fig. 1b. Here we focus on the red curve that represents the lowest order even mode of the waveguide and lies within the bandgap defined by the blue curves and below the light line in black. Fig. $1 \mathrm{~b}$ shows that the bandwidth over which guidance can be obtained is only $0.78 \%$ corresponding to $12 \mathrm{~nm}$ at a central wavelength of $1550 \mathrm{~nm}$. This is too small and will result in significant leakage of energy to the light line. As a result, the Q-factor of any resonant cavity would be small.
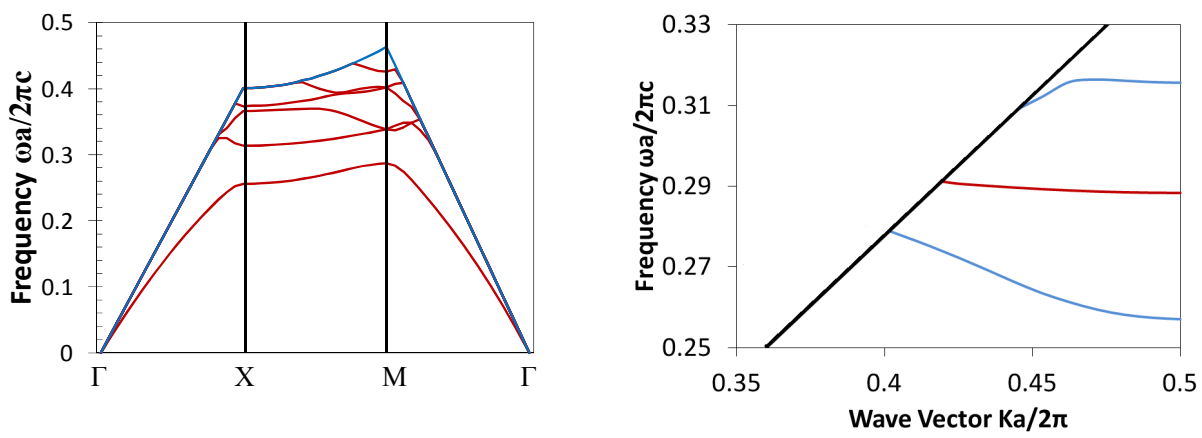

Fig.1a: Calculated band structure for a $\mathrm{Ge}_{11.5} \mathrm{As}_{24} \mathrm{Se}_{64.5}$ photonic crystal with index 2.65 fully embedded in a silica cladding with index 1.44. 1b. Guided modes of a W1 waveguide (red) embedded in the PhC lattice of Fig 1a. 
In order to create a high-Q resonant cavity within an embedded structure, we need to find a method to broaden the bandwidth of the guided mode of the photonic crystal waveguide. After many cycles of calculations, we found that if the waveguide width is reduced down to W0.52 W0.58, the bandwidth for the guided even mode increased substantially as shown in Fig. 2a. W0.58, W0.56, W0.54 and W0.52 waveguides in fact exhibit bandwidths of $2.8 \%, 2.5 \%, 2.1 \%$ and $1.8 \%$ respectively which corresponds to values ranging from $28 \mathrm{~nm}$ to $43 \mathrm{~nm}$ which proves to be sufficient to prevent the light from leaking to the light line and potentially allows high-Q to be achieved.
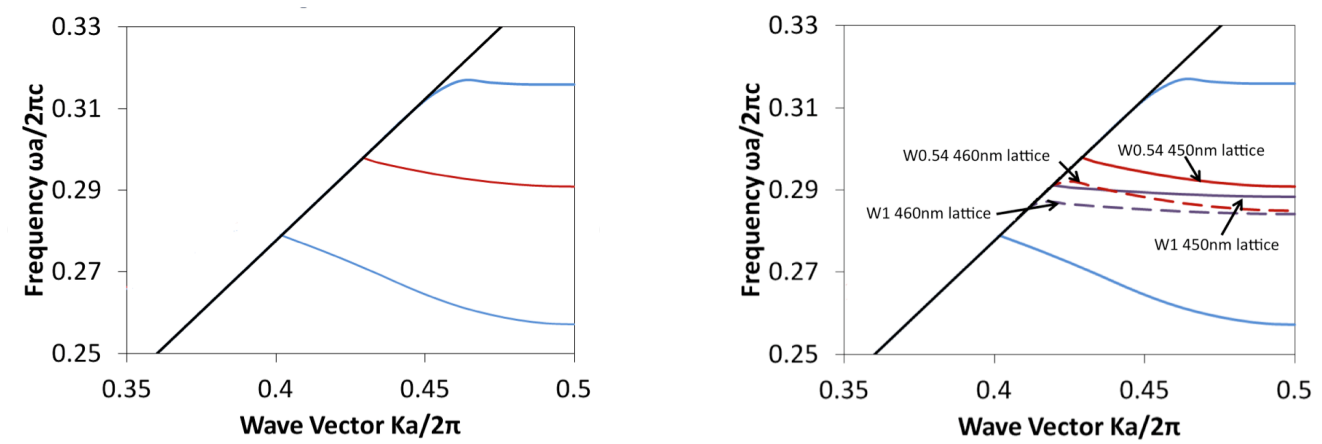

Fig.2a: Guided modes of a W0.54 waveguide (red) embedded in the PhC lattice of Fig 1a. Fig. 2b, a comparison of the dispersion for the modes of both W1 and W0.54 waveguides that demonstrates the increased bandwidth achieved using the W0.54 structure. The dispersion curves are shown for two structures whose lattice constant differs by $10 \mathrm{~nm}$.

Combined with a narrow photonic crystal waveguide, a hetero-structure resonant cavity gives us the opportunity to create a high Q-factor cavity by utilizing the gap between two guided modes of different waveguide produced by varying the lattice constant along the waveguide. Fig. $2 \mathrm{~b}$ shows the guided mode of two waveguides with two different lattice constants and waveguide widths. One thing that needs to be mentioned is that in a hetero-structure, the photonic crystal with the smaller lattice constant is used as the mirror. This is because there is a portion of bandwidth of guided mode in the larger lattice that does not exist in the smaller one. As a result, a gap between the two guided modes is formed which defines the frequency range where a resonance can be located. Fig. $2 b$ shows the guided modes for a W1 waveguide for two lattice constants differing by $10 \mathrm{~nm}$. In this case the gap between the two curves exceeds the bandwidth over which guidance occurs. Thus the modes of the waveguide with the larger lattice can couple to the light line and this results in serious leakage of energy into the cladding. As a result, the resonant cavity cannot achieve high Q.

In the narrow W0.54 waveguide, the bandwidth of the guided mode is about three times broader than that of the W1 waveguide and the gap is now completely located inside the bandwidth over which guidance occurs. Therefore, there is no extra leakage into the light line and a high $\mathrm{Q}$ factor should be achievable in this structure.

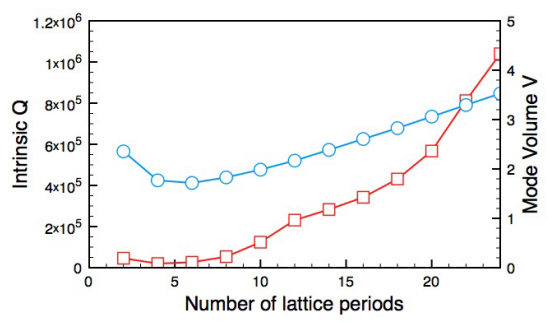

Fig 3: Variation of Q (red) and mode volume V (blue) as a function of cavity length for a W0.54 waveguide and heterostructure with a stepped mirror

In addition to the Q-factor, the effective mode volume $V_{\text {eff }}$ is another important parameter for a resonant cavity. It defines the volume over which the resonance extends and determines the light intensity inside the cavity and the minimum power required to exciting switching. The effective volume is often represented in terms of the mode volume where 
$V=n^{3} V_{\text {eff }} / \lambda^{3}$ where $\lambda$ is the wavelength of resonance and $n$ the refractive index of the material. As both Q-factor and $V$ determine the light intensity resonating in the cavity, $\mathrm{Q} / V$ is routinely used to quantify resonant cavities.

Fig. 3 plots the Q-factor and $V$ as a function of cavity length (in lattice periods). Both Q-factor and mode volume first decrease between 2 to 6 lattice periods and then start to rise. For 24 lattice periods the Q factor can be over 1,000,000 with an effective mode volume of 3.5. This relatively small volume is achieved because the resonant cavity is formed from a very narrow waveguide even though the cavity is quite long. Thus with this design the mode volume can remain reasonably small.

So far we have discussed the intrinsic Q-factor of the resonator, $\mathrm{Q}_{v}$, which is achieved with complete confinement by semi-infinite mirrors at each end of the cavity. However, in a real device, the mirror reflectivity needs to be reduced to allow energy to be coupled in and out of the cavity. As a result, a lower Q-factor, $\mathrm{Q}_{\text {total }}$, is determined by the coupling efficiency in and out of the cavity and this can be represented as below:

$$
\begin{gathered}
T=\frac{\left(Q_{v}-Q_{\text {total }}\right)^{2}}{\left(Q_{v}\right)^{2}}, \\
T=\left(\frac{Q_{\text {total }}}{Q_{v}}\right)^{2},
\end{gathered}
$$

for end-coupled and side-coupled cavities respectively.

In order to design our photonic crystals, we calculated the $\mathrm{Q}_{\text {total }}$ and $T$ for mirrors extending over different numbers of lattice periods for a 24 period long cavity with $\mathrm{Q}_{v}$ over $1,000,000$. $\mathrm{Q}_{\text {total }}$ increases as more lattice periods are added to the mirrors whilst the transmission decreases. With mirrors 14 lattice periods long, $\mathrm{Q}_{\text {total }}$ is almost equal to $\mathrm{Q}_{v}$ but the transmission has dropped almost to zero. As a result, we need an even higher intrinsic $\mathrm{Q}_{v}$ to compensate the cost of mirror transmission. A graded mirror structure can achieve this enhancing the mirror reflectivity by smoothing the effective index change at the edge of the cavity. As is well known, this enhances the intrinsic Q-factor of hetero-structure micro-resonators. Introducing a graded mirror consisting of a single section two lattice periods long with a period intermediate between the cavity and the mirror, the $\mathrm{Q}$ factor could be increased to 2,500,000. By using two intermediate sections each two lattice periods long, the $\mathrm{Q}$ was further enhanced to over 4,000,000. Furthermore, by reducing the lattice constant difference between the mirror and cavity from $10 \mathrm{~nm}$ to $4 \mathrm{~nm}$, a Q factor $>20,000,000$ is achievable according to calculation using a 24 period long cavity with two intermediate sections.

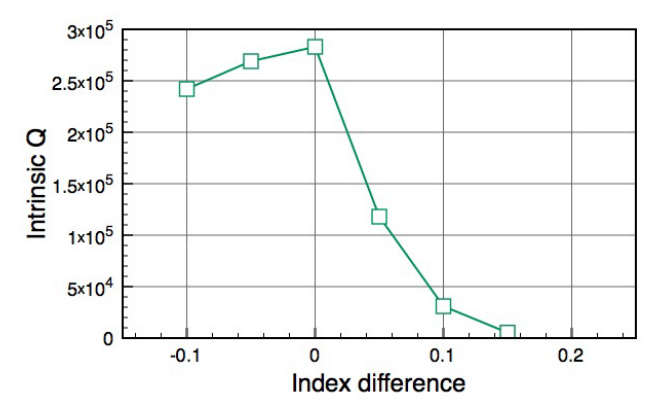

Fig 4. Predicted variation of Q-factor as a function of the mismatch in index between the top and bottom claddings

Another important property of a fully clad resonant cavity is its resistance to refractive index mismatch between the silica substrate and the material used as a top cladding. Since it is difficult to fill the small holes of the PhC with silica, we intended to use either a UV-curable polymer coating applied as a liquid or a refractive index matching oil as the top cladding. As a result, the refractive index of the top cladding might differ from that of the silica substrate and it is important to know what refractive index mismatch can be tolerated without seriously compromising the achievable Q. The results are shown in Fig. 4 that demonstrates that a positive refractive index mismatch leads a dramatic reduction of 
the Q-factor basically because of enhanced coupling to the light line. However, if the refractive index of the top cladding is smaller than the design, the structure is quite robust against an index mismatch.

\section{FABRICATION AND CHARACTERIZATION}

A photonic crystal conforming to the above designs was fabricated using the following process. Firstly, a $\mathrm{Ge}_{11.5} \mathrm{As}_{24} \mathrm{Se}_{64.5}$ film was thermal deposited onto an oxidized silicon wafer as a substrate. Then, about 100nm of PMMA was spin-coated onto the film as a positive resist for EBL. The photonic crystal was patterned into the PMMA using a Raith 150 electron beam lithography tool and wet developed. Inductively coupled plasma etching using $\mathrm{CHF}_{3}$ gas was then used to transfer the photonic crystal into the $\mathrm{Ge}_{11.5} \mathrm{As}_{24} \mathrm{Se}_{64.5}$ glass. After etching and removal of the PMMA, a $2 \mathrm{~nm}$ thick layer of $\mathrm{Al}_{2} \mathrm{O}_{3}$ was deposited by atomic layer deposition as a protective coating. According to the SEM images the resulting photonic crystal was of high quality with vertically etched surfaces.

After hand cleaving, a Cargille refractive liquid with and index of 1.45 (at 589nm) was added as a top cladding. The structure consisted of a resonator 8 lattice periods long that had a $+6 \mathrm{~nm}$ increase in its lattice constant relative to the mirrors. A graded mirror was employed consisting of two sections with their lattice periods offset by $-2 \mathrm{~nm}$ and $-4 \mathrm{~nm}$ relatively to the resonator. For this structure the intrinsic Q-factor was predicted to be $\approx 2,000,000$. In order to make it easier to measure the intrinsic Q-factor, we included a side-coupled resonator in the design (as well as the in-line endcoupled resonator) so that Eq. $1 \mathrm{~b}$ could be used to deduce $\mathrm{Q}_{v}$.
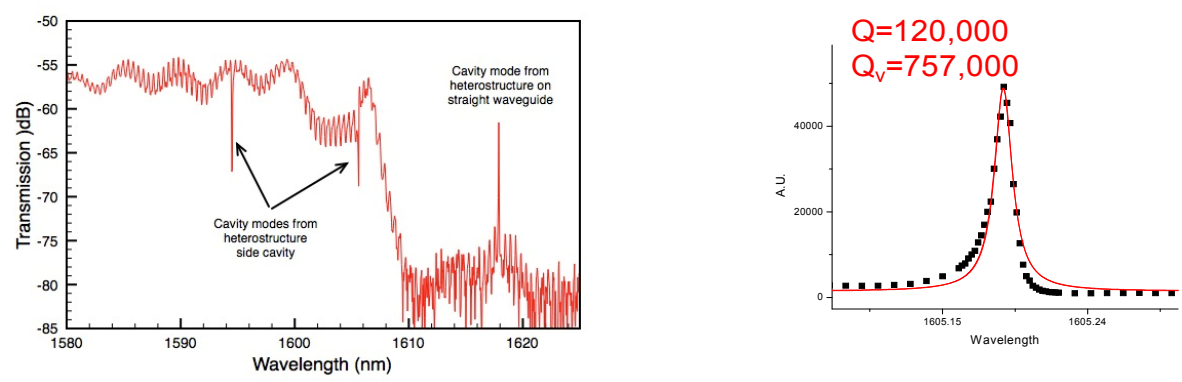

Fig. 5a. Optical transmission of fully embedded $\mathrm{Ge}_{11.5} \mathrm{As}_{24} \mathrm{Se}_{64.5} \mathrm{PhC}$ measured using a supercontinuum source and OSA with a resolution of $0.01 \mathrm{~nm}$. Three resonance peaks are apparent: two from the side cavity at $\approx 1594 \mathrm{~nm}$ and $1605 \mathrm{~nm}$ and one from the in-line cavity at $\approx 1618 \mathrm{mn}$. $5 \mathrm{~b}$ : a scan of the $1605 \mathrm{~nm}$ resonance using a tunable laser with $1 \mathrm{pm}$ resolution fitted to a Lorentzian curve. From this measurements the intrinsic Q can be deduced to be 757,000.

We initially probed the transmission of the $\mathrm{PhC}$ with a super-continuum spectrum as a broadband light source measuring the transmission with an optical spectrum analyzer with a resolution of $0.01 \mathrm{~nm}$ (Fig 5a) in order to locate the cavity resonances. Three resonances were apparent: those at $1594 \mathrm{~nm}$ and $1605 \mathrm{~nm}$ were due to the side-coupled cavity whilst that at $1618 \mathrm{~nm}$ was the in-line cavity. The drop in transmission at $1607 \mathrm{~nm}$ is due to the band edge of the mirrors in the in-line cavity. The loaded Q-factor for the cavity at $1605.2 \mathrm{~nm}$ was measured to be 120,000 using a tunable CW laser with a resolution of $1 \mathrm{pm}$. This resonance is shown (inverted) in Fig. $5 \mathrm{~b}$ and was associated with a dip in the transmission $-16 \mathrm{~dB}$ deep and a width of $13.3 \mathrm{pm}$. The spectrum was fitted with a Lorenzian to obtain the spectral width and by applying Eq. 5.2 the intrinsic Q-factor could then be calculated to be 757,000. This is the highest value ever reported for a chalcogenide photonic crystal with the intrinsic $\mathrm{Q}_{v}$ rivaling what has been achieved in silicon PhCs [19].

\section{CONCLUSIONS}

We have shown that with careful design, high-Q micro-cavities can be produced in chalcogenide glass $\mathrm{PhC}$ fully embedded in a silica cladding in spite of the low index contrast of only 1.2. Using a narrow W0.52-W0.58 waveguide extends the bandwidth of the guided mode within the bandgap strongly reducing coupling to the light line that inevitably occurs in the case of a W1 waveguide limiting its Q. Using a W0.54 waveguide design intrinsic $\mathrm{Q}_{\nu} \mathrm{s}$ of $>10^{7}$ are possible in ideal structures. To demonstrate the design we fabricated a hetero-structure cavity with graded mirrors to a design that that had a calculated intrinsic $\mathrm{Q}_{v}$ of 2,000,000. We determined the width and depth of a resonance for a loaded cavity 
side-coupled to a bus waveguide to be 120,000 and $-16 \mathrm{~dB}$ respectively. From these we could determine the $\mathrm{Q}_{v}$ of the fabricated resonator to be $\approx 757,000$. The difference between the measured and predicted values is almost certainly attributable to deviations in the structure from the ideal. By increasing the mirror transmission this would be sufficient to achieve loaded Q's of $\approx 10,000$ with a resonance $>30 \mathrm{~dB}$ deep. This should support bi-stable Kerr switching on a timescale of several $10 \mathrm{~s}$ ps. Finally a real benefit of the embedded structure is its mechanical stability and the potential for a-thermal behavior. In the present structure the thermo-optic coefficient of the oil used as a cladding was large and negative and in fact reversed the temperature dependence of the resonance frequency relative to the unclad glass. This suggests that zero temperature dependence is achievable if a suitable cladding can be found.

\section{REFERENCES}

[1] Johnson, S. G., Fan, S., Villeneuve, P. R., Joannopoulos, J. D., and Kolodziejski, L. A., "Guided modes in photonic crystal slabs," Phys. Rev. B 60(8), 5751-5758 (1999).

[2] Johnson, S. G., Villeneuve, P. R., Fan, S., and Joannopoulos, J. D., "Linear waveguides in photonic-crystal slabs," Phys. Rev. B 62(12), 8212-8222 (2000).

[3] Baba, T., "Slow light in photonic crystals", Nature Photonics 2, 465 - 473 (2008)

[4] Baba T. and Mori, D., "Slow light engineering in photonic crystals", J. Phys. D: Appl. Phys. 402659 (2007)

[5] de Sterke, C. M., Walker, J., Dossou, K. B., and Botten, L. C., "Efficient slow light coupling into photonic crystals," Opt. Express 15, 10984-10990 (2007)

[6] Baba, T., "Toward Photonic Crystal Optical Buffer," in Conference on Lasers and Electro-Optics/Quantum Electronics and Laser Science Conference and Photonic Applications Systems Technologies, OSA Technical Digest (CD) (Optical Society of America, 2008), paper CWH1.

[7] Burmeister, E. F., Mack, J. P., Poulsen, H.N., Mašanovic, M. L., Stamenic, B., Blumenthal, D. J., and Bowers, J. E., "Photonic integrated circuit optical buffer for packet-switched networks," Opt. Express 17, 6629-6635 (2009)

[8] Zhai, Y., Tian, H., and Ji, Y., "Slow Light Property Improvement and Optical Buffer Capability in Ring-Shape-Hole Photonic Crystal Waveguide," J. Lightwave Technol. 29, 3083-3090 (2011)

[9] Hao, R., Cassan, E., Kurt, H., Le Roux, X., Marris-Morini, D., Vivien, L., Wu, H., Zhou, Z., and Zhang, X., "Novel slow light waveguide with controllable delay-bandwidth product and utra-low dispersion," Opt. Express 18, 5942-5950 (2010)

[10] Monat, C., Spurny, M., Grillet, C., O’Faolain, L., Krauss, T. F., Eggleton, B. J., Bulla, D., Madden, S., and LutherDavies, B., "Third-harmonic generation in slow-light chalcogenide glass photonic crystal waveguides," Opt. Lett. 36, 2818-2820 (2011)

[11] Corcoran, B., Monat, C., Grillet, C., Moss, D. J., Eggleton, B. J., White, T. P., O'Faolain L., and Krauss, T. F., "Green light emission in silicon through slow-light enhanced third-harmonic generation in photonic-crystal waveguides", Nature Photonics 3, 206 - 210 (2009)

[12] Monat, C., Grillet, C., Corcoran, B., Moss, D. J., Eggleton, B. J., White, T. P., and Krauss, T. F., "Investigation of phase matching for third-harmonic generation in silicon slow light photonic crystal waveguides using Fourier optics," Opt. Express 18, 6831-6840 (2010)

[13] Oda, H., Inoue K., Tanaka, Y., Sugimoto, Y., Ishikawa, H., and Asakawa, K., "Self-phase modulation in photoniccrystal-slab line-defect waveguides," Appl. Phys. Lett. 90, 231102:1-3 (2007)

[14] Suzuki, K., Hamachi, Y., and Baba, T., "Fabrication and characterization of chalcogenide glass photonic crystal waveguides," Opt. Express 17(25), 22393-22400 (2009).

[15] McMillan, J. F., Yu, M., Kwong, D-L, and Wong, C. W., "Observation of four-wave mixing in slow-light silicon photonic crystal waveguides," Opt. Express 18, 15484-15497 (2010)

[16] Monat, C., Ebnali-Heidari, M., Grillet, C., Corcoran, B., Eggleton, B. J., White, T. P., O’Faolain, L., Li, J. and Krauss, T. F., "Four-wave mixing in slow light engineered silicon photonic crystal waveguides," Opt. Express 18, 22915-22927 (2010)

[17] Eckhouse, V., Cestier, I., Eisenstein, G., Combrié, S., Colman, P., De Rossi, A., Santagiustina, M., Someda, C. G. and Vadalà, G., " Highly efficient four wave mixing in GaInP photonic crystal waveguides," Opt. Lett. 35, 1440-1442 (2010) 
[18] Akahane, Y., Asano, T., Song, B. and Noda, S., "High-Q photonic nanocavity in a two-dimensional photonic crystal," Nature 425, 944-947 (2003).

[19] Song, B., Noda, S., Asano, T., and Akahane, Y., "Ultrahigh-Q photonic double heterostructure nanocavity," Nat. Mater. 4, 207-210 (2005)

[20] Akahane, Y., Asano, T., Song, B-S., and Noda, S., "Fine-tuned high-Q photonic-crystal nanocavity," Opt. Express 13, 1202-1214 (2005)

[21]Lee, M. W., Grillet, C., Monat, C., Mägi, E., Tomljenovic-Hanic, S., Gai, X., Madden, S., Choi, D-Y., Bulla, D., Luther-Davies, B., and Eggleton, B. J., "Photosensitive and thermal nonlinear effects in chalcogenide photonic crystal cavities," Opt. Express 18, 26695-26703 (2010)

[22] Notomi, M., Shinya, A., Mitsugi, S., Kira, G., Kuramochi, E., and Tanabe, T., "Optical bistable switching action of Si high-Q photonic-crystal nanocavities," Opt. Express 13, 2678-2687 (2005)

[23] Tanabe, T., Notomi, M., Mitsugi, S., Shinya, A., and Kuramochi, E., "Fast bistable all-optical switch and memory on a silicon photonic crystal on-chip," Opt. Lett. 30, 2575-2577 (2005).

[24] Kim. M.-K., Hwang, I.-K., Kim, S.-H., Chang, H.-J., and Lee, Y.-H., "All-optical bistable switching in curved microfiber-coupled photonic crystal resonators," Appl. Phys. Lett. 90,161118 (2007).

\section{ACKNOWLEDGEMENTS}

The research was conducted by the Australian Research Council Centre for Excellence for Ultrahigh bandwidth Devices for Optical Systems (project number CE110001018) 